quer à l'aide de l'équation (1): l'augmentation de la concentration $b$ entrâne une réduction de la vitesse du front d'oxydation dans un rapport assez faible comparé à l'augmentation de la température d'oxydation qui donne une croissance exponentielle du coefficient de diffusion.

Le fait important sur l'alliage $\mathrm{Cu}-\mathrm{Ti}$ est le mélange de deux oxydes qui se traduit par deux séries d'anneaux en microdiffraction électronique et deux rayons de particules en diffusion centrale des rayons $X$, ce qui rend parfois assez délicat le dépouillement des courbes de diffusion. La comparaison des intensités intégrées expérimentales et théoriques rend compte de la transformation $\mathrm{TiO}_{2}$ anatase en $\mathrm{TiO}_{2}$ rutile avec une décroissance de $Q_{0}$ à $1000^{\circ} \mathrm{C}$ et des particules d'oxyde beaucoup plus grosses.

Mais on a toujours un grand écart entre $Q_{0}$ expérimental et $Q$ théorique de l'anatase pour les températures inférieures ou égales à $900^{\circ} \mathrm{C}$. Cet écart est du même ordre de grandeur que celui observé pour $\mathrm{Cu}-\mathrm{Be}$; or, si pour ce dernier alliage on peut supposer l'existence d'un oxyde mixte qui relâche les contraintes et favorise la cohérence observée, il n'en est pas de même pour les particules d'oxyde de titane qui sont incohérentes. Il faut alors admettre qu'une partie non négligeable du titane reste en solution dans l'alliage. Du point de vue thermodynamique, il est logique de penser qu il reste plus de titane que de béryllium dans le cuivre car l'oxyde de titane est moins stable que l'oxyde de béryllium; l'influence de la température d'oxydation et du recuit le prouve. Par conséquent, la dissolution de $\mathrm{TiO}_{2}$ est plus importante aux températures élevées de l'oxydation.

Cette étude par diffusion centrale des rayons $X$ nous a permis de suivre l'influence de la température d'oxydation, d'un recuit ultérieur et de la pression d'oxygène. paramètres liés à la taille et à la nature des particules d’oxyde formées au sein des alliages $\mathrm{Cu}-\mathrm{Be}$ et $\mathrm{C} 1 \mathrm{Ti}$ qui subissent l'oxydation interne.

Liée à d'autres méthodes d'investigation, en particulier la microscopie électronique, la diffusion centrale se révèle une méthode de choix, bien adaptée à l'étude de petites particules d'oxyde. On peut en effet mettre en évidence de faibles variations sur les tailles des particules et sur leurs formes. La comparaison entre la mesure de l'intensité totale diffusée et sa valeur théorique nous donne des renseignements importants sur la nature de l'oxyde formé et sur les transformations qui peuvent se produire à l'intérieur de l'alliage.

\section{Références}

Ashby, M. F. \& Brown, L. M. (1963). Phil. Mag. 8, 16491676.

BöHM, G. \& Kahlweit, M. (1964). Acta Met. 12, 641-648. Guinier, A. (1939). Ann. Phys. 12, 162-237.

Guinier, A. \& Fournet, G. (1955). Small-Angle Scattering of $X$-rays. New York: John Wiley.

HirschHORN, J. S. \& LeNel, F. V. (1966). Trans. A.S.M. 59, 208-221.

HUCKEl, W. (1951). Structural Chemistry of Inorgani: Compounds. Amsterdam: Elsevier.

JONES, R. L. \& KELLY, A. (1968). Oxide Dispersion Strengthching. pp. 229-252. Edité par G. S. ANSFLL.. New York: Gordon and Breach.

Komatsu, N. \& Grant, N. J. (1962). Trans. AIME, 224, 705713.

MilJERINGi, J. L. (1971). Adranc. Mater. Res. 5, 1-75.

Maudon, A., Allain, J. \& Maruin, P. (1973). Mem. Sci. Rer. Metall. JXX (2), 165-169.

NaUdon. A. \& Caisso, J. (1972). Scripta Met. 6, 59-63.

Naudon, A. \& (aisso, J. (1974). J. Appl. Cryst. 7, 000-000.

Nauion, A. \& Jaulin, M. (1968). Rer. Phys. Appl. 3, $152-$ 156.

Poidiurski, H. H. \& Davis, F. N. (1964). Trans. AIME, $230,731-738$.

Soul.́, J. L. (1957). J. Phys. Radium, 18, 90A-102A.

Spengier, H. (1965). Metall, 19, 725-728.

Tohoubar, D. \& Mering, J. (1969). J. Appl. Ciyst. 2, 128138.

Wiritums, D. M. \& Smin, G. C. (1968). Oxide Dispersion Strengthening pp. 509. 536. Fdité par G. S. AnsfLL. New York: Gordon and Breach.

WoOlnousf, G. R. \& Brow', L. M. (1970). J. Inst. Met. 98. $106-110$.

\title{
X-ray Small-Angle Scattering Measurements on Metallic Catalysts
}

\author{
By A. Renouprez and P. Bosch-Giral \\ Institut de Recherche sur la catalyse, 39 Boulerard du II Novembre 1918, 69100-Villeurbanne, France
}

The granulometry of a metallic phase, dispersed on the surface of porous or non-porous supports was determined. Reliable results were obtained on samples containing a metal amount as low as $0.5 \%$. Measurements are easier with non-forous supports; however, in the case of microporous supports, a special technique was used to introduce the 'pore maskant' even in the finest micropores (20 $\AA$ in diameter). For ultrafine particles $(10-30 \AA)$ the results are in good agreement with electron microscope data. When some larger particles are present in the sample, the distribution curves are broad and the small-angle method leads to more reproducible results than electron microscopy. 